\title{
Procedures for Fertility Preservation in Female Cancer Patients
}

\author{
ZOLTÁN LANGMÁR ${ }^{1,2}$, KÁLMÁN IVÁNYI', FERENC BÁNHIDY', \\ LÁSZLÓ TORGYÍK², MAGDOLNA DANK²
}

\author{
${ }^{1} 2^{\text {nd }}$ Department of Obstetrics and Gynecology, Faculty of Medicine, \\ Semmelweis University, Budapest, ${ }^{2}$ Department of Radiology and Oncotherapy, \\ Faculty of Medicine, Semmelweis University, Budapest, \\ ${ }^{3}$ Faculty of Medicine, Semmelweis University, Budapest, Hungary
}

\begin{abstract}
Improving survival rates and quality of life following modern combined cancer treatments have resulted in a growing number of patients requesting maintenance of reproductive functions. Few methods are currently available to maintain fertility during oncotherapy. Even though most of them are still experimental and their efficacy and safety have not been determined, the future for fertility preservation in women with cancer is promising. In vitro fertilization with embryo cryopreservation offers an established method, but it could be risky to delay cancer treatment regarding the progression of several cancer types. Moreover, exposure to a high estrogen milieu during ovarian stimulation is undesirable when patients have estrogen sensitive malignant tumors. Cryopreservation of mature oocytes following in vitro fertilization and intracytoplasmatic sperm injection offers advantages, but it is still limited due to its low success rate. Emerging techniques of ovarian tissue cryopreservation followed by autotransplantation have been clinically explored. Novel technologies of tissue freezing and thawing promise improving results. This procedure can be offered in the future for prepubertal girls before cancer treatment to maintain future fertility. Gonadal tissue cryopreservation and transplantation should be considered experimental in humans for the present time until greater evidence regarding efficacy and safety is accrued.
\end{abstract}

Keywords: fertility preservation, chemotherapy, radiotherapy, cryopreservation

Modern anticancer drugs and novel oncotherapeutic methods have resulted in increasingly better survival rates and quality of life. However, this therapeutic success has been accompanied by the loss of fertility and premature menopause in many women cured of their disease. Consequently, efforts to preserve reproductive functions are of increasing importance [1-6]. The significance of such research is further emphasized by current statistical data showing that approximately one in 700 adults is a survivor of a certain childhood malignant disease. It is estimated that by the year 2010 this proportion will significantly increase and almost one in 250 adults will suffer from the late negative consequences of treatments of childhood cancer, includ-

Corresponding address: Zoltán Langmár MD, Üllői út 78/a, H-1082 Budapest, Hungary.

E-mail: langmarzoltan@hotmail.com 
ing fertility problems, and, in severe cases, infertility [5-6]. There are promising methods for fertility preservation in patients treated for malignant diseases, but most of them are still on experimental level or studied in clinical trials [5-7]. When choosing a method, the age, sex, patients' general condition and long-term prognosis of the malignant disease should be taken into consideration [5-6]. This requires a close cooperation of the representatives of subdisciplines involved in the treatment of such patients (oncohematologist, paediatrician, gynaecologist, experts of assisted reproduction and psychologists). In addition, patients (in the case of children their relatives) should be educated on the technical details and informed about the benefits, efficacy and potential risks of the feasible methods based on the available data. It should also be kept in mind that some procedures (e.g. hormonal stimulation prior to oocyte aspiration) are time-consuming and consequently may delay the start of the definitive oncological treatment and, therefore, negatively affect the chance for therapeutic success. Consequently, such procedures can be recommended only after careful evaluation of the clinical situation $[1,5-8]$. The techniques of most of the feasible assisted reproduction methods (e.g. cryopreservation of embryos) are well established, while other procedures, like cryopreservation and later transplantation of oocytes and ovarian tissue are still in an experimental phase. Therefore, these latter procedures can be used only in controlled studies supervised by ethical committees, after careful evaluation of the potential risks and benefits $[5-6,9]$. A special evaluation is required by the fact that the risk of retransmission of tumor cells and consequently the risk of recurrence in association with transplantation of ovarian tissue is not completely known $[1,5-7,10-11]$. Treatments resulting in high estrogen levels (controlled ovarian stimulation with gonadotropin prior to oocyte aspiration) can be associated with a more pronounced, accelerated progression of some estrogen-dependent malignancies. Therefore, these methods in such cases are not recommended [5]. If ovarian stimulation is required in breast cancer patients, it is more sensible to use tamoxifen to stimulate ovaries. Alternatively, aromatase inhibitors appear to be useful. In this publication we review from the point of view of the oncological practice the preferable methods of preservation of female fertility which are either currently available or hopefully will be used safely and successfully in the near future. In certain gynecological tumours, patients may benefit from fertility-sparing surgery (the uterus and contralateral ovary are preserved). Ovarian tumours of young females usually have a low risk of bilateral involvement, thus sparing the contralateral normal ovary and uterus may be feasible. This is especially feasible in the treatment of borderline ovarian tumours, sex cord stromal tumours, and germ cell tumours of the ovary. The promising surgical procedure, trachelectomy (abdominal or vaginal), developed partially by Hungarian gyn-oncologists (Ungár et al.), can preserve future fertility in certain stages of cervical cancer [12].

\section{Effects of Oncotherapy on Fertility}

It is well known that both cytotoxic-cytostatic treatments and radiation therapy have a negative impact on the reproductive function of the ovaries. The impairment can be partial, or complete and irreversible. These processes usually result in premature (early) menopause and fertility problems or infertility. The lack of production of sex hormones has other severe consequences including osteoporosis, increased cardiovascular risk, and psychosomatic symptoms [1, 5-6, 9, 13-14]. Studies in cancer survivors performed up to now have not demonstrated significantly increased occurrence of genetic impairments $[8,31]$. However, when pregnant, these patients definitely require closer attention and follow-up from the obstetrical point of view, too. Comparable stud- 
ies evaluating the post-treatment fertility of cohorts of female cancer survivors are rare. Indirect assessment of biochemichal markers of ovarian function can provide evidence of partial loss of the ovarian reserve. However, these approaches do not assist to predict as to how many female cancer survivors will suffer infertility [2].

\section{Radiotherapy}

Human gametes are extremely sensitive to ionizing radiation. This effect is especially strong and irreversible in less-differentiated cells (oogonium and spermatogonium). The degree and persistence of the damage of gametes caused by radiation depend on the cumulative dose of irradiation, the location and size of the target area to be treated and the age of the patient. In older patients more serious damage is to be expected. High doses of radiation are mutagenic, embryotoxic and teratogenic. In addition, the blood supply of the cervical muscle and the whole uterus should also be taken into consideration [5-7, 15]. It has been reported in many studies that in pregnancies of patients who had previously received radiation therapy there was an increased occurrence of spontaneous abortion, premature delivery and low birth weight. Cranial irradiation used in the treatment of central nervous system tumours has a negative impact on fertility due to disruption of the hypothalamus-hypophysis-ovary axis. The ovaries are exposed to significant doses of radiation in the treatment of cervical and rectal cancer [5]. Thibaud et al. demonstrated that whole-body irradiation used in prepubertal age resulted in a complete ovarian failure in $55-80 \%$ of patients. Salooja et al., studying the ability of getting pregnant of adults receiving whole-body radiation therapy in childhood, reported a less than $3 \%$ pregnancy rate [17].

\section{Bone Marrow Transplantation}

In the last 30 years, bone marrow transplantation has become an effective therapy of several malignant hematological diseases. Therapeutic protocols are composed of high dose chemotherapy with or without radiation therapy, followed by stem cell transplantation $[1,5]$. In a recent study the pregnancy rate of 38,000 patients has been investigated after childhood bone marrow transplantation. Only 129 pregnancies were reported in the study group [13]. This extraordinarily low pregnancy rate was demonstrated in other studies, too $[1,8]$.

\section{Chemotherapy}

The severity of damage of gametes following cytotoxic treatments depends on the age, the applied agents and the cumulative doses of drugs. Many chemotherapeutic agents have gonadotoxic effects (Table 1). Generally, it is very hard to precisely calculate or predict for any medicine the level of damage it inflicts, since chemotherapeutics are used usually in combinations. However, the impairment of the ovaries and the loss of reproductive function are well-known complications of chemotherapy [1, 4-5, 13]. The most severe gametotoxicity is caused by alkylating agents (cyclophosphamide, chlorambucil), but antimetabolites (methotrexate, 5-fluorouracil, cytosine arabinoside, gemcitabine) are also known for their significant gametotoxic potential [5]. Byrne et al. followed patients receiving chemotherapy in childhood for 20 years 
Table 1 Risk of gonadotoxicity of various antineoplastic drugs (after Wallace et al.)

\begin{tabular}{lll}
\hline High risk & Moderate risk & Low risk \\
\hline Cyclophosphamide & Cisplatin & Vincristine \\
Ifosfamide & Carboplatin & Methotrexate \\
Busulphan & Doxorubicin & Dactinomycin \\
Melphalan & Gemcitabine & Bleomycin \\
Procarbazine & & Mercaptopurine \\
Chlorambucil & & Vinblastine \\
\hline
\end{tabular}

and observed early menopause (up to 31 years of age) at $42 \%$ of these patients [13]. In these patients ovarian failure, therefore the lack of sex hormone production is associated with risks of several other pathological conditions (osteoporosis, increased risk of cardiovascular diseases, psychological disorders) [5]. Clinical evaluation is especially complicated by the fact that preservation of reproductive function is not necessarily predicted by post-treatment restoration of regular periods [5-6]. Therefore, young patients intending to have children are advised to become pregnant after the end of treatments followed by an at least 12 month-long disease-free period, within a couple of years, since they have to take into consideration the potential development of early menopause and the consequent infertility. At an older age (above 38-40 years) a greater proportion of ovarian failure is to be expected, since young patients still have significantly more primordial follicle reserve $[5-7,14]$.

\section{Fertility Preservation Strategies}

Surgical and assisted reproductive methods are available to preserve fertility for the post-oncotherapy period, but most of them are still in experimental phase. In prepubertal age only cryopreservation and later transplantation of ovarian tissue seems to be feasible, but in young patients of reproductive age other methods can also be used for preservation of fertility $[4-6,14]$.

\section{Ovarian Transposition (Oophoropexy)}

Pelvic irradiation is used in the treatment of several malignancies. As described above, pelvic radiotherapy is gametotoxic and results in ovarian failure. In order to avoid that, McCall et al. were the first to perform ovarian transposition. Currently this intervention is recommended to be performed laparoscopically which is a minimal-invasive and safe method. During oophoropexy ovaries (with preservation of their blood supply) are transposed out of the target area of radiation therapy. This method may preserve fertility, since ovarian dose can be reduced to $5-10 \%$ of untransposed ovaries, minimizing the level of impairment. The procedure is not completely riskfree and can be associated with consecutive chronic pelvic pain, infarction of the fallopian tubes, and formation of ovarian cysts. It has been suggested that the ovaries be transposed at least $3 \mathrm{~cm}$ from the upper border of the radiation field, but overstretching the infundibulopelvic ligament may result in compromised ovarian blood flow or adnexal torsion. Furthermore, its value is limited to young cancer patients who are treated with radiation only [18]. 


\section{Cryopreservation of Fertilized Oocytes}

Cryopreservation, storage and post-recovery implantation of embryos are well-known, widely used assisted reproduction procedures with a well-established technique $[1,14]$. There are sophisticated freezing-thawing protocols in place to ensure efficacy. This is by far the most efficient cryobanking option. In principle, viability of frozen embryos can be preserved indefinitely, making implantation at a later date possible. An additional advantage of cryopreservation is that it leaves sufficient time to perform tests for preimplantation genetic diagnostics. However, a disadvantage of cryopreservation is that prior to obtaining oocytes, controlled ovarian hyperstimulation is necessary which requires $2-6$ weeks of time $[1,5,7]$. Consequently, oncotherapy can be started only with delay which may be highly unfavourable for the patient. This method should not be used in prepubertal age and in female patients without partner rejecting even the use of donor sperm. In addition, treatments resulting in high estrogen levels may increase the progression of some hormone-dependent tumours (e.g. breast cancer, endometrial cancer). In such cases the administration of tamoxifen or aromatase inhibitors (letrozole) administered simultaneously with gonadotropins is preferred, but the clinical experience is not unambiguous. Many ethical and legal questions can be raised in connection with this method, because there is no clear position on what to do with the frozen embryos if the patient dies. Despite the problems cryopreservation of the embryos is a well-established method of assisted reproduction. Therefore, it has a stable place in fertility preservation ensuring the ability of getting pregnant in the postrecovery lifetime of cancer patients $[1,5-6,14]$.

\section{Oocyte Cryopreservation}

Cryopreservation is a promising method for storage of oocytes until later use for in vitro fertilization. Despite the fact that sperm cryopreservation has been available for decades, deep freezing of oocytes until now has not become a routine procedure $[5,14,19-20]$. At the moment, this is far less efficient than the cryopreservation of fertilized eggs. With few exceptions, this procedure is in the case-report mode. The first child from a cryopreserved oocyte was born in 1986. There are 200-250 such pregnancies or births worldwide [20-22]. There are many technical problems to face when using this method. Oocytes are very sensitive to low temperatures due to their water content, big size and low volume-surface ratio [4, 20-21]. Ice crystal formation and depolymerization damage of the mitotic spindle (which plays a central role in cell division) should be taken into consideration despite data from the latest studies showing that the mitotic spindle is much more resistant to freezing than previously thought. Damage of the mitotic spindle results in a more frequent occurrence of aneuploidy. As a consequence of the above-mentioned processes a hardening of zona pellucida may occur, although this problem can be solved by using intracytoplasmatic sperm injection (ICSI) [20-23]. There is a significant difference between the sensitivity of mature and premature oocytes to freezing process. Premature oocytes have a significantly better survival rate after the cryopreservation-thawing procedure, but, as a disadvantage, injury of the cell nucleus and aneuploidy may occur more frequently. A serious problem with premature oocytes is that after thawing only a few oocytes can be made eligible for fertilization. Mature (metaphase stage II) oocytes are especially sensitive to low temperatures [20, 22-23]. Special preservative solution and freezing procedures have been developed to improve the results. Progress in this area concerns the use of microinjection, changing medium composition (by substituting 
choline for sodium), and using stronger concentrations of extracellular cryoprotectants such as sucrose during the cooling and especially the rewarming stages [3]. For a long period of time a method of slow freezing and rapid thawing proposed by Chen et al. has been used, but currently better results were achieved by the development of novel cryoprotective solutions and the vitrification method aimed to prevent ice crystal-formation. Vitrification is a freezing procedure that traps all of the aqeous solutions in a solid phase, described as amorphous or vitreous, preventing ice-crystal formation. Unlike classical slow-cooling protocols, vitrification avoids cellular lesions caused by extracellular ice crystals. The cryoprotectant concentration needs to be kept as low as possible, for reasons of cytotoxicity, while being high enough to ensure a critical cooling speed [3, 23-25]. It has been demonstrated that the survival rate of oocytes following this method is about 64-69\%. As mentioned above, efficacy can be further improved by using intracytoplasmatic sperm injection [23]. In summary, more clinical studies are needed to improve this method, since current pregnancy rates are much lower than those reported after cryopreservation of the embryo. However, this method is associated with much less ethical and legal problems, since it does not require any partner or donor sperm and is acceptable for those who have religion-based objections against cryopreservation of embryos [4-5, 20, 23]. New technologies used during freezing and thawing result are currently resulting in more and more improving oocyte survival rates and hopefully increasingly better pregnancy rates. Porcu et al. reported a $14 \%$ live birth rate following oocyte cryopreservation, in vitro fertilization by ICSI and implantation of the fertilized oocyte. These procedures have but a modest clinical efficiency [23]. Further disadvantages of this method are that cancer patients may not have more than one opportunity for oocyte harvesting before undergoing potentially sterilizing treatment, since a cycle of controlled stimulation requires several weeks, and there is a delay before cancer treatment cycles [5].

\section{The Role of GnRH Analogues}

The efficacy of GnRH analogues in preservation of gonadal functions has not been established yet. Neither benefits nor long-term consequences were unambiguously determined with these preparations; therefore, they can be used for such purposes only in controlled, prospective clinical studies. The protective effect of $\mathrm{GnRH}$ preparations against gonadotoxicity is proposed based on their known effects. During treatments with GnRH analogues lower gonadotropin concentrations, as a consequence of low estrogen level, reduced ovarian perfusion, and taken altogether suppression of ovarian function were observed. Nevertheless, the safety of using GnRH analogues in conjunction with chemotherapy has not been clearly established. Moreover, not only are $\mathrm{GnRH}$ analogues are expensive and cause severe menopausal symptoms, but the long-term use may also be associated with irreversible bone loss $[5,18]$.

\section{Cryopreservation of Ovarian Tissue}

During this procedure storage of thousands of primordial follicles can be managed and there is no need for prior ovarian stimulation. Since primordial follicles are located in the cortical tissue of the ovary, a biopsy from cortical strips is enough [5, 26-27]. This intervention may take place during laparotomy due to tumour removal, or, if no such intervention is indicated, in association with laparoscopy, always before pharmacotherapy or radiation therapy. Premature oocytes are 
smaller, characterized by a lower metabolic ratio. Due to lack of some anatomical structures they are significantly more resistant to freezing than mature oocytes [22, 27]. Deep freezed tissues can be autotransplanted after thawing or "cultured" aiming maturation of primordial follicles and premature oocytes. The first report on storage of cryopreserved living human cells was published in 1948. In 1994 restoration of fertility was observed in experiments with sheep after autotransplantation of ovarian tissue [25]. Ten years later, in 2004 the first child was born after autotransplantation of ovarian tissue to a mother who had previously received aggressive chemotherapy due to stage IV Hodgkin's disease. Before treatment, five biopsy samples were removed from the left ovary. At age 32, after chemotherapy - which caused the patient to be amenorrheic - the thawed fragments of ovary were returned to the ovary. Periods resumed, and 9 months after the transplantation, a spontaneous pregnancy occurred. A healthy girl was born at term [28]. However, some critical remarks have been made on the work of Donnez et al., because there is no unambiguous evidence whether the fertilized oocyte comes really from the transplanted ovarian tissue or from the remaining ovaries [5, 14, 29-30].

As long as there is no contraindication, ovarian tissue is collected via laparoscopy. In paediatric age groups, a large cortical biopsy may be enough since their ovaries harbor a larger number of follicles than the adult ovary. Autotransplantation of ovarian tissue may be conducted in the pelvis, near to the original location of the ovary (orthotopic transplantation). Using sharp and blunt dissection, a pocket is created in the ovarian fossa, posterior to the broad ligament, superior to the ureters, and inferior to iliac vessels in the supine position. Transplantation can be done using a heterotopic approach also (e.g. under the skin of the forearm or abdomen). An advantage of this latter method can be a better control of the function of the ovarian tissue. In addition, obtaining oocytes for in vitro fertilization is significantly simpler $[10,30]$. The operation is done under local anesthesia and follow-up is easier with heterotopic transplantation. As cryopreservation of ovarian tissue is available in more and more centers, compilation of widely accepted clinical guidelines by assisted reproduction experts on the principles of use of this method (in what cases can or should this method be used) is becoming more important. It should be emphasized that this method is still in the experimental phase; therefore it can be used only in patients with an expected long-term survival after recovery from cancer $[6-7,10]$. At this publication, it would seem that oocyte cryopreservation is more efficient than ovarian tissue cryopreservation if preembryo storage is not feasible.

\section{Safety of Cryopreservation of Ovarian Tissue - the Problem of Potential Reimplantation of Cancer Cells}

Altough ovarian tissue cryopreservation may be the only acceptable method for any prepubertal or premenarchal female patients receiving chemotherapy or radiation therapy, the safety of autotransplantation of ovarian tissue is of key importance in cancer patients [5]. The risk of potential reimplantation of cancer cells needs careful evaluation. Before the start of treatment of nonHodgkin lymphoma, Kim et al. performed xenotransplantation of human ovarian tissue in experimental animals. They could not demonstrate any recurrence of lymphoma [29]. Despite all of these observations this could not be unambiguously demonstrated in other tumour types. Therefore, this method can be used in the future only after careful oncological-oncopathological consultation, following the evaluation of risks and potential benefits, on the personal request of 
the patient. A histological assessment of micrometastases should therefore always be carried out on a small portion of the harvested tissue prior to cryopreservation. Special considerations are required especially in haematological or lymphatic malignancies ("sui generis" systemic malignant diseases). However, this method is strictly contraindicated in patients with tumours frequently metastasizing in the ovary (advanced, stage IV or lobular breast cancer, cervical adenocarcinoma, neuroblastoma) or in primary ovarian carcinoma $[5,10]$. Prior to autotransplantation of ovarian tissue a detailed pathological examination (including immunohistochemical methods, flow cytometry, molecular genetic techniques) is necessary. In summary, despite the safety concerns this is a highly promising method, because it can be performed prior to childhood oncological treatments ensuring later fertility of the patient [10].

\section{Perspectives of Cryopreservation Research of Ovarian Tissues}

One of the future methods can be the removal, cryopreservation and later autotransplantation of the whole ovary with vascular anastomosis preventing ischaemic injury of the ovarian tissue. In this intervention the biggest problem is constituted not by the surgical technique but the technique itself of cryopreservation of the complete ovary $[5,10,31]$. Endothelial cells are more sensitive to freezing, which can be associated with irreversible organ damage and necrosis [32]. Currently only the rat studies produced success [33]. Ice crystal formation, mentioned above, and problems with penetration of cryoprotective agents plays here a role, too [32]. Vitrification seems preferable for cryopreserving more complex and heterogeneous biological systems such as whole ovaries [18]. One possibility would be to vitrify the whole ovaries with their lumbo-aortic vascular pedicle so as to reduce follicle loss on graft revascularisation. It has been demonstrated that primordial follicles are much more sensitive to ischaemia than to freezing and thawing. Theoretically, ischaemic injury may be prevented by antioxidants (vitamin E, superoxide dismutase, ascorbic acid) or increased expression of antiapoptotic and angiogenesis stimulating angiogenetic factors. Many antiangiogenetic factors (VEGF, TGF, angiopoietin) stimulating angiogenesis are known. Indirectly, gonadotrop hormones also stimulate angiogenesis $[5,10,30]$.

\section{Conclusions}

Before initiating oncological treatments associated with an increased risk of fertility problems, there are a few methods available to patients to preserve fertility $[3,5,10,30]$. Certain sophisticated assisted reproduction procedures (cryopreservation and later implantation of fertilized oocytes) are technically well established but some of their aspects are associated with an increased risk to recovery from the malignant disease, since they may delay the start of the actual oncological therapy, while in the case of estrogen dependent tumours progression of the tumour may be accelerated. The advantage of cryopreservation of mature oocytes is that this method does not require any partner or donor sperm. However, this method also includes stimulation treatment which may cause a delay in starting oncological treatment. Unfortunately the success rate of this method is still very low. The most promising method is cryopreservation of the ovarian tissue which can revolutionize fertility preservation possibly in the near future, when and if technical problems are solved. Ovarian cryopreservation is also ethically challenging. This strategy is 
potentially useful, but it is too early to determine its true benefits. In contrast to oocyte or embryo freezing, ovarian tissue cryopreservation does not delay cancer treatment. It can be offered even to prepubertal girls and would restore ovarian function. However, it requires surgery. The age limit for the procedure is debatable, but it may not be justified to collect ovarian tissue from infants or very young girls whose ovaries are small and for whom surgery is a high risk. It can be more problematic in minors. Young female cancer patients are still being poorly counseled with regard to the negative impact of the treatment on their fertility and on their options for fertility preservation. It should be emphasized that these methods are still in experimental phase and can be used only in a controlled environment, in special studies, meeting strict ethical and legal preconditions, reviewed by institutional and national ethical boards. Many cancer patients can be mentally more vulnerable and desperate when they realize that they may be infertile after oncotherapy. They should be well informed to prevent false expectations as well as the possibility of being exploited by the commercial interests of the purveyors of unproven technologies $[2,5-6,10,20]$.

\section{References}

[1] Huser, M., Jurankova, E., Crha, I.: Fertility preservation strategies in women undergoing chemotherapy for haematological malignancy. Eur. Clin. Obstet. Gynecol., 2006, 2, 77-81.

[2] Pacey, A. A.: Fertility issues in survivors from adolescent cancers. Cancer Treat. Rev., 2007, 33, 646-655.

[3] Lornage, J., Salle, B.: Ovarian and oocyte cryopreservation. Curr. Opin. Obstet. Gynecol., 2007, 19, 390-394.

[4] Konc, J., Kanyó, K., Cseh, S.: Deep freezing of oocytes for preserving the fertility of young female oncology patients treated with aggressive radio- and/or chemotherapy. (In Hungarian) Orv. Hetil., 2006, 147, 655-658.

[5] Maltaris, T., Seufert, R., Fischl, F. et al.: The effect of cancer treatment on female fertility and strategies for preserving fertility. Eur. J. Obstet. Gynecol. Reprod. Biol., 2007, 130, 148-155.

[6] Wallace, H. W. B, Anderson, R., Irvine, D. S.: Fertility preservation for young patients with cancer: who is at risk and what can be offered? Lancet Oncol., 2005, 6, 209-218.

[7] Weintraub, M., Gross, E., Kadari, A. et al.: Should ovarian cryopreservation be offered to girls with cancer. Pediatr. Blood Cancer, 2007, 48, 4-9.

[8] Bath, L. E., Hamish, W., Wallace, B.: Late effects of the treatment of childhood cancer on the female reproductive system and the potential for fertility preservation. Br. J. Obstet. Gynecol., 2002, 109, 107-114.

[9] Ginsberg, J. P., Womer, R. B.: Preventing organ-specific chemotherapy toxicity. Eur. J. Cancer, 2005, 41, 2690-2700.

[10] Oktay, K., Buyuk, E.: Ovarian transplantation in humans: indications, techniques and the risk of reseeding cancer. Eur. J. Obstet. Gynecol. Reprod. Biol., 2004, 113S, S45-S47.

[11] Radford, J.: Autotransplantation of ovarian tissue and the risk of disease transmission. Eur. J. Obstet. Gynecol. Reprod. Biol., 2004, 113S, S48-S49.

[12] Ungár, L., Pálfalvi, L., Hogg, R., Siklós, P. et al.: Abdominal radical trachelectomy: a fertility-preserving option for women with early cervical cancer. Br. J. Obstet. Gynecol., 2005, 112, 366-369.

[13] Byrne, J.: Infertility and premature menopause in childhood cancer survivors. Med. Pediat. Oncol., 1999, 33, 24-28.

[14] Oktay, K.: An individualized approach to fertility preservation in women with cancer. J. Supportive Oncol., 2006, 4, 181-183.

[15] Lo Presti, A., Ruvolo, G., Gancitano, R. A. et al.: Ovarian function following radiation and chemotherapy for cancer. Eur. J. Obstet. Gynecol. Reprod. Biol., 2004, 113, S33-S40. 
[16] Thibaud, E., Rodriguez-Macias, K., Trivin, C. et al.: Ovarian function after bone marrow transplantation during childhood. Bone Marrow Transplant., 1998, 21, 287-290.

[17] Salooja, N., Szydlo, R. M., Socie, G. et al.: Pregnancy outcomes after periferial blood or bone marrow transplantation. Lancet, 2001, 358, 271-276.

[18] Oktay, K., Sönmezer, M.: Fertility preservation in gynecologic cancers. Curr. Opin. Oncol., 2007, 19, 506-511.

[19] Jain, J. K., Paulson, R. J.: Oocyte cryopreservation. Fertil. Steril., 2006, 86, 1037-1046.

[20] Kim, S. S.: Fertility preservation in female cancer patients: current developments and future directions. Fertil. Steril., 2006, 85, 1-11.

[21] Porcu, E.: Oocyte freezing. Sem. Reprod. Med., 2001, 19, 221-230.

[22] Porcu, E., Fabbri, R., Damiano, G. et al.: Oocyte cryopreservation in oncological patients. Eur. J. Obstet. Gynecol. Reprod. Biol., 2004, 5, S14-16.

[23] Porcu, E., Venturoli, S.: Progress with oocyte cryopreservation. Curr. Opin. Obstet. Gynecol., 2006, 18, 273-279.

[24] Chen, C.: Pregnancy after human oocyte cryopreservation. Lancet, 1986, 1, 884-886.

[25] Tucker, M., Morton, P., Liebermann, J.: Human oocyte cryopreservation: a valid alternative to embryo cryopreservation. Eur. J. Obstet. Gynecol. Reprod. Biol., 2004, 113S, S24-S27.

[26] Gook, D. A., Edgar, D. H., Stern, C.: Cryopreservation of human ovarian tissue. Eur. J. Obstet. Gynecol. Reprod. Biol., 2004, 113S, S41-S44.

[27] Gook, D. A., Osborn, S. M., Archer, J. et al.: Follicle development following cryopreservation of human ovarian tissue. Eur. J. Obstet. Gynecol. Reprod. Biol., 2004, 113S, S60-S62.

[28] Donnez, J., Dolmans, M. M., Demylle, D. et al.: Livebirth after orthotopic transplantation of cryopreserved ovarian tissue. Lancet, 2004, 364, 1405-1410.

[29] Kim, S. S., Hwang, In-Tak, Lee, H. C.: Heterotopic autotransplantation of cryobanked human ovarian tissue as a strategy to restore ovarian function. Fertil. Steril., 2004, 82, 930-932.

[30] Oktay, K., Buyuk, E., Veeck, L. et al.: Embryo development after heterotopic transplantation of cryopreserved ovarian tissue. Lancet, 2004, 363, 837-840.

[31] Imhof, M., Bergmeister, H., Lipovac, M. et al.: Orthotopic microvascular reanastomosis of whole cryopreserved ovine ovaries resulting in pregnancy and live birth. Fertil. Steril., 2006, 85 (Suppl. 1), 1208-1215.

[32] Woods, E. J., Benson, J. D., Agca, Y.: Fundamental cryobiology of reproductive cells and tissues. Cryobiology, 2004, 48, 146-156.

[33] Courbiere, B., Odagescu, V., Baudot, A. et al.: Cryopreservation of the ovary by vitrification as an alternative to slow-cooling protocols. Fertil. Steril., 2006, 86 (Suppl. 3), 1243-1251. 